Aporte Santiaguino 11(1), enero-junio 2018: 11-20

ISSN-L 2616-9541

\title{
Análisis de función y matriz morfológica aplicado al diseño de un implemento agrícola
}

\author{
Analysis of function and morphological matrix applied to the design of an \\ agricultural implement
}

\author{
Raúl A. Dean ${ }^{1}$, Livio S. Maglione ${ }^{1}$ y Fernando O. Cappellari ${ }^{1}$
}

\section{RESUMEN}

El objetivo de la investigación fue explorar y generar diseños alternativos de un implemento para aplicaciones agrícolas, particularmente, de un sistema móvil de bombeo de aguas poco profundas de ríos, lagos y canales, aplicando métodos de diseño apropiados a una etapa de diseño conceptual. Como implemento de una máquina agrícola, es de especial relevancia para realizar riego complementario, así como para contribuir al drenaje en zonas de inundación. El método de análisis de función y la matriz morfológica forman parte de la metodología que se utilizó. Se resume la fase inicial del diseño conceptual en la que fueron aplicados ambos métodos, logrando identificar combinaciones factibles de configuraciones del sistema.

Palabras clave: proceso de desarrollo de producto; análisis de función; matriz morfológica.

\section{ABSTRACT}

The objective of the present research was to explore and generate alternative designs of the application of an agricultural implement, particularly, of a mobile system for pumping shallow water from rivers, lakes and canals, applying appropriate design methods to a conceptual design stage. As an implement of an agricultural machinery, it is of special relevance to perform complementary irrigation, as well as to contribute

\footnotetext{
${ }^{1}$ Universidad Nacional de Río Cuarto. Río Cuarto, Argentina.

(C) Los autores. Este artículo es publicado por la Revista Aporte Santiaguino de la Universidad Nacional Santiago Antúnez de Mayolo. Este es un artículo de acceso abierto, distribuido bajo los términos de la Licencia Creative Commons Atribución-NoComercial-CompartirIgual 4.0 Internacional. (http://creativecommons.org/licenses/ by-nc-sa/4.0/), que permite el uso no comercial, distribución y reproducción en cualquier medio, siempre que la obra original sea debidamente citada.
} 
to drainage in flood zones. The method of function analysis and morphological matrix part of the methodology that was employed. The initial phase of the conceptual design in which both methods were applied is summarized, being able to identify feasible combinations of system configurations.

Keywords: product development process; function analysis; morphological matrix.

\section{INTRODUCCIÓN}

Una de las problemáticas de la producción agrícola de la región centro sur de la provincia de Córdoba (Argentina) es la mala distribución temporal de las precipitaciones y la ocurrencia de escasez de las mismas. El riego complementario es una práctica disponible que permite subsanar esta adversidad y estabilizar los rendimientos de los cultivos extensivos característicos de la zona. El área bajo riego por aspersión en Argentina está muy por debajo del total cultivado posible de regar, si bien creció a un ritmo considerable hasta el año 2000 hoy se encuentra estabilizado. La provincia de Córdoba posee un buen potencial de riego, definido por la gran superficie de tierras aptas, que coinciden con zonas donde es posible obtener aguas subterráneas en cantidad y calidad suficiente y se complementa con las importantes fuentes de agua superficial. La condición agro-climática determina que, con oportunos agregados de agua, es posible obtener altos rendimientos en forma estable en el tiempo (Salinas et al., 2006).

De esta problemática surge una oportunidad de mejora si se supone que el potencial hidráulico de aguas superficiales puede ser aprovechado racionalmente con fines de riego.

Este trabajo tiene como objetivo presentar la fase de diseño conceptual de un sistema de bombeo cuando el agua proviene de una fuente superficial poco profunda $(\leq 40$ $\mathrm{cm}$ ) y su uso para fines agrícolas no es continuo, como en el caso de tener que suministrar agua con fines de riego solo en períodos específicos de crecimiento de cultivos.

\section{MATERIALES Y MÉTODOS}

La presente es una investigación típica de las ciencias de lo artificial, la cual tiene el propósito de generar objetos, dispositivos, herramientas, sistemas artificiales que aún no existen, o adaptar o modificar aquellos existentes para lograr mejores resultados $\mathrm{u}$ otras funcionalidades.

Una ciencia del diseño tiene objetivos específicos; busca generar artefactos de diferentes naturalezas para la solución de problemas (o "clases problemáticas"). Una ciencia del diseño no es "ciencia aplicada". Su propósito no es la mera traducción en la práctica de los enunciados explicativos de las ciencias fácticas, sino la formulación y validación de las reglas de diseño -concepción, diseño e implementación en circunstancias definidas- que serán impulsadas por los profesionales del campo específico cuando los juzguen pertinente (Dresch et al., 2015, 16). 
El foco está en las soluciones propuestas y el objetivo principal de investigación es prescribir cursos de acción para lograr los estados deseados. La cuestión típica de investigación son las soluciones alternativas para una clase dada de problemas.

En la fase inicial del proceso de diseño son objetivos particulares la definición de una función técnica y su análisis en subfunciones, requerimientos, atributos, generación de alternativas y selección, ello en el marco de un diseño conceptual del sistema, de manera de satisfacer requerimientos impuestos por entradas, morfología del terreno, interfaces con la máquina motriz y el potencial uso del sistema.

Resultado de esta investigación es la obtención de un conocimiento de diseño conceptual que habilita la posterior continuidad del diseño en detalle, prototipado y prueba del implemento agrícola en su función técnica asignada.

Para la fase del diseño conceptual se utiliza una formulación básica de análisis de función y síntesis morfológica para la obtención de una configuración cinemática de un mecanismo aplicado a un sistema de bombeo de aguas superficiales.

\section{Análisis de función}

Con el método de análisis de función se propone inicialmente la función técnica asignada al sistema artificial en términos de la conversión que se debe realizar sobre un conjunto de entradas para obtener el conjunto de salidas deseadas, en otras palabras, "qué va a ser logrado por el sistema diseñado y no sobre cómo va a ser logrado (Cross, 1996)".

El análisis de función comienza con la descripción de la función técnica asignada al sistema que resuelve el problema técnico. El problema técnico es captar y transportar agua desde un punto de toma ubicado al nivel de aguas poco profundas hasta otro nivel, alejado del primero horizontalmente y con una mayor altura correspondiente con el perfil de desnivel del terreno, debiendo vencer en este transporte las resistencias de conducción, desnivel y acondicionando la cantidad y calidad del agua con valores provenientes del diseño agronómico e hidráulico para que, en su etapa de salida, pueda ser utilizada por un equipo de riego para producir, por ejemplo, una lluvia artificial.

La función técnica de bombeo (figura 1), puede ser implementada transmitiendo y/o transformando movimientos, energía, fuerzas e información para posicionar una bomba hidráulica y su sistema de captación de agua, ponerla en funcionamiento, operarla, conducir el fluido y disponerlo con los valores hidráulicos (h), por ejemplo caudal total y presión de diseño del sistema de riego de aspersión, y físicos (f) adecuados en la entrada de otro equipo que realizará la aplicación agrícola correspondiente. Es requisito lograr la salida deseada a través de una transformación del estado no satisfaciente con el soporte de entradas normalizadas y accesibles en la interface tractor - implemento. 


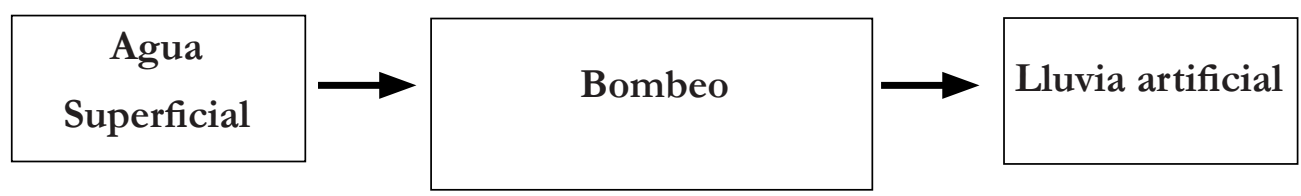

Figura 1. Función técnica

Usualmente la transformación es una tarea compleja que requiere identificar diferentes subfunciones equivalentes en su conjunto a la función técnica (figura 2). Este análisis convierte la caja negra de la función técnica en una transparente donde cada sub función (SF) podrá ser materializada mediante diferentes dispositivos o mecanismos coetáneos al diseño del medio artificial.
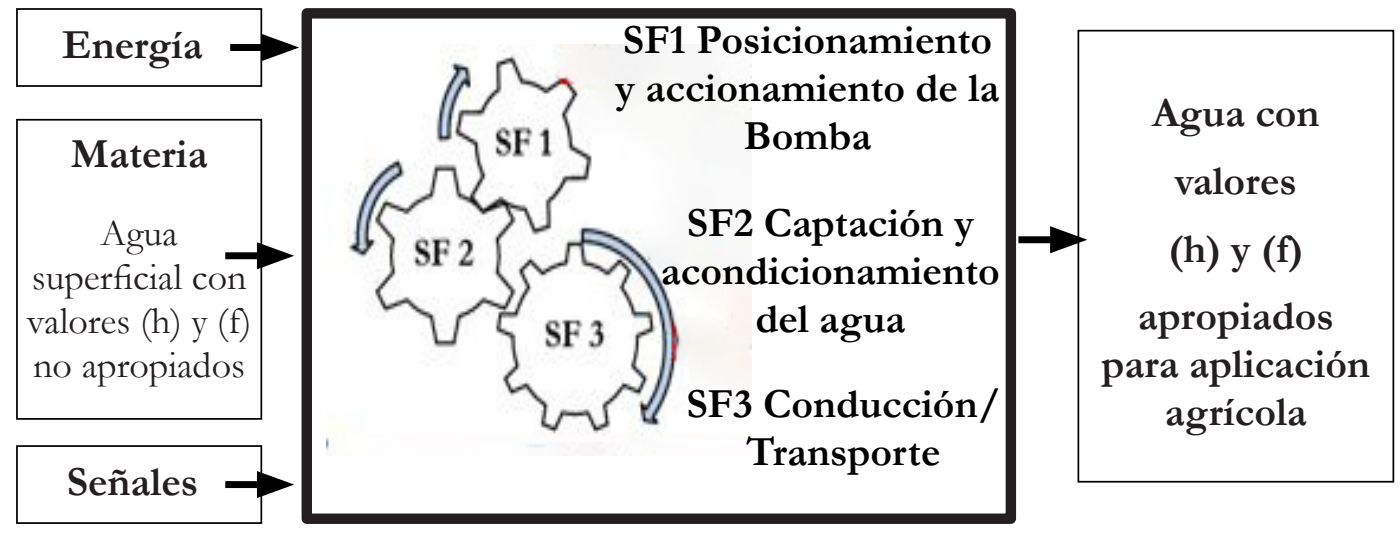

ENTRADAS SUBFUNCIONES - MEDIO ARTIFICIAL

SALIDAS

Figura 2. Subfunciones (SF) - Implemento Agrícola Móvil de Bombeo

Los requerimientos están relacionados con la geometría, cinemática, fuerzas, energía, material, señales, seguridad, ergonomía, economía, ecología, entre otros. En lo que respecta a energía sus requerimientos son: fuente normalizada (eléctrica/hidráulica) para el funcionamiento del mecanismo y del sistema de bombeo, con determinados valores de eficiencia, presión, voltaje, caudal, almacenaje, capacidad, etc; con respecto a material con: flujo y transporte, valores mínimos y máximos de propiedades físicas y químicas, equipos auxiliares, etc; en lo que respecta a señales con: entradas y salidas, interface visual, sistema de control, etc.

\section{Análisis morfológico}

La definición general de morfología es "el estudio de la forma o patrón”, es decir, la forma y la disposición de las partes de un objeto, y cómo estos se "conforman" para crear un conjunto. Esencialmente, es un método para identificar e investigar un conjunto de posibles soluciones ("configuraciones") para un dado problema complejo. 
El análisis morfológico está basado sobre los siguientes supuestos (Finger, 1989a): 1. cualquier problema complejo de ingeniería puede ser dividido en un número finito de subproblemas, 2. cada uno de los subproblemas puede ser considerado separadamente y sus relaciones con otros subproblemas temporariamente suspendidas, 3. todos los subproblemas y sus soluciones pueden ser presentadas en una tabla morfológica, 4. una solución global para cualquier problema complejo de ingeniería puede ser encontrado como una combinación de soluciones a subproblemas individuales, 5. una solución global puede ser encontrada en una forma imparcial a través de una generación aleatoria de combinaciones de soluciones a subproblemas desde la tabla morfológica.

Entre los modelos prescriptivos de diseño, el análisis morfológico ha sido exitosamente utilizado en el diseño de configuraciones (Finger, 1989b; Cross, 1996).

La lista de atributos es un buen punto de partida para métodos analítico-combinatorios, tales como el análisis morfológico. Distintos atributos del implemento surgen de la información morfométrica el terreno donde se utiliza. Se considera un área de cultivo en cercanías de un río o arroyo con un perfil típico de desnivel. (figura 3) La forma del perfil representa sus características geométricas a excepción de su posición y orientación.

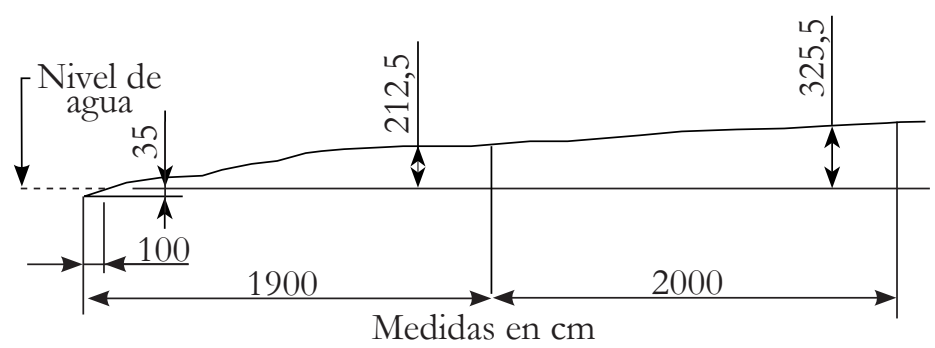

Figura 3. Perfil altimétrico del terreno

El sistema debe ser de geometría variable para poder adaptarse al mismo y a otros perfiles similares, estable, de fácil transporte, liviano, y poder ser implementado a distintas plantas motrices (tractores) que se disponen en el ámbito rural. Este último requisito se fundamenta en que su implementación debe considerar tanto los sistemas normalizados de la máquina motriz agrícola, tractor, tales como el enganche en tres puntos y las tomas de potencia del mismo, como el presupuesto de capital que debe afrontar un productor agropecuario que decide invertir en sistemas de riego artificial. Además, debe poder ser utilizado como un sistema de rápida actuación para el bombeo de aguas en zonas anegadas por problemas de inundaciones. En contribución a la conservación del medio ambiente, la propuesta de diseño es la de un implemento agrícola ecológico puesto que no se plantea ningún tipo de intervención a los cursos de agua que implique modificar la topografía, por lo que el diseño del sistema móvil de bombeo, deberá 
superar los problemas relacionados a la poca profundidad de los ríos del sur de la provincia de Córdoba, Argentina.

Un método heurístico adicional que contribuye a la investigación de la morfología del implemento es la analogía. Kapp (1877) conjeturó que: “una herramienta es tanto más fácily cómoda de manejar cuanto más influida está en su forma por la proporción orgánica modélica". Sobre la base de este supuesto, la configuración cinemática de un sistema móvil de bombeo de aguas superficiales acoplado a una máquina agrícola puede ser pensada considerando la anatomía de un brazo, un dedo o una mano humana, en lo que respecta a poder lograr el posicionamiento y manipulación de la carga; en tal sentido configuraciones factibles de un mecanismo móvil para un sistema de bombeo pueden ser consideradas similares a las configuraciones cinemáticas adoptadas para robots industriales.

El objetivo del método es generar un conjunto de soluciones alternativas de diseño para un producto, y por lo tanto ampliar la investigación para nuevas soluciones potenciales. Para el análisis de la morfología del sistema móvil sobre una base analógica se consideraron configuraciones básicas de mecanismos coetáneos de un brazo robótico poliarticulado.

Tabla 1. Carta o matriz morfológica

\begin{tabular}{|c|c|c|c|c|c|}
\hline Soluciones & 1 & 2 & 3 & 4 & 5 \\
\hline $\begin{array}{c}\text { Configuración } \\
\text { básica }\end{array}$ & Cartesiana & Cilíndrica & $\begin{array}{l}\text { Esférica o } \\
\text { polar }\end{array}$ & $\begin{array}{c}\text { Brazo } \\
\text { articulado }\end{array}$ & $\begin{array}{l}\text { Angular o } \\
\text { antropomórfica }\end{array}$ \\
\hline $\begin{array}{l}\text { Fuente de } \\
\text { energía }\end{array}$ & Mecánica & Hidráulica & Eléctrica & & \\
\hline Actuador & Neumático & Hidráulico & Eléctrico & & \\
\hline $\begin{array}{l}\text { Transporte de } \\
\text { materia (agua) }\end{array}$ & $\begin{array}{l}\text { Tubería } \\
\text { metálica }\end{array}$ & $\begin{array}{l}\text { Tubería } \\
\text { PVC }\end{array}$ & & & \\
\hline $\begin{array}{l}\text { Sensor / } \\
\text { Control }\end{array}$ & Posición & Cinemático & Dinámico & Adaptativo & \\
\hline $\begin{array}{l}\text { Sistema de } \\
\text { control }\end{array}$ & $\begin{array}{l}\text { Inteligencia } \\
\text { artificial }\end{array}$ & $\begin{array}{l}\text { Operador } \\
\text { humano }\end{array}$ & $\begin{array}{l}\text { Servo } \\
\text { Sistemas }\end{array}$ & & \\
\hline $\begin{array}{l}\text { Manipulador } \\
\text { de carga }\end{array}$ & $\begin{array}{l}\text { Garra de } \\
\text { fricción }\end{array}$ & $\begin{array}{l}\text { Garra con } \\
\text { forma }\end{array}$ & $\begin{array}{l}\text { Sujección } \\
\text { semi } \\
\text { permanente }\end{array}$ & & \\
\hline
\end{tabular}




\section{RESULTADOS}

Como modelo simplificado de mecanismo plano se adopta, con criterios adicionales, la solución inferida desde la carta morfológica y que se esquematiza en la figura 4. La movilidad se consigue mediante una secuencia en serie de dos barras articuladas por una junta y un actuador o cilindro hidráulico que gobierna los movimientos relativos de ambas barras, repitiéndose este esquema dos veces en su montaje, estando las barras indicadas de 1 a 4 . La barra 1 constituye el sistema de anclaje, la barra 2 el brazo inferior, la barra 3 el brazo central, la barra 4 el brazo superior y el soporte 5 constituye el soporte vertical del sistema de bombeo. La cinemática generada por el accionamiento de tres cilindros hidráulicos C1, C2 y C3 articulados entre dos barras consecutivas, 1-2, 2-3 y 3-4, permite el posicionamiento del brazo 5 que soporta el módulo de bombeo. El brazo 5 trabaja en forma vertical, por gravedad, y es donde estará montada la bomba hidráulica. El manipulador es de cadena cinemática abierta al tener dos eslabones unitarios colocados en sus extremos, mientras que los eslabones internos son binarios.

La bomba es la unidad básica del sistema y su trabajo consiste en aspirar el agua desde la fuente de abastecimiento de aguas poco profundas, y conducirla hasta el cuerpo de la misma donde se genera la presión necesaria para elevarla venciendo las pérdidas de carga y la necesaria para el trabajo de los aspersores. Es fundamental una adecuada elección y diseño de la bomba a utilizar, puesto que debe entregar el caudal de agua requerido por la demanda hídrica y a una presión determinada para el correcto funcionamiento del equipo de riego.

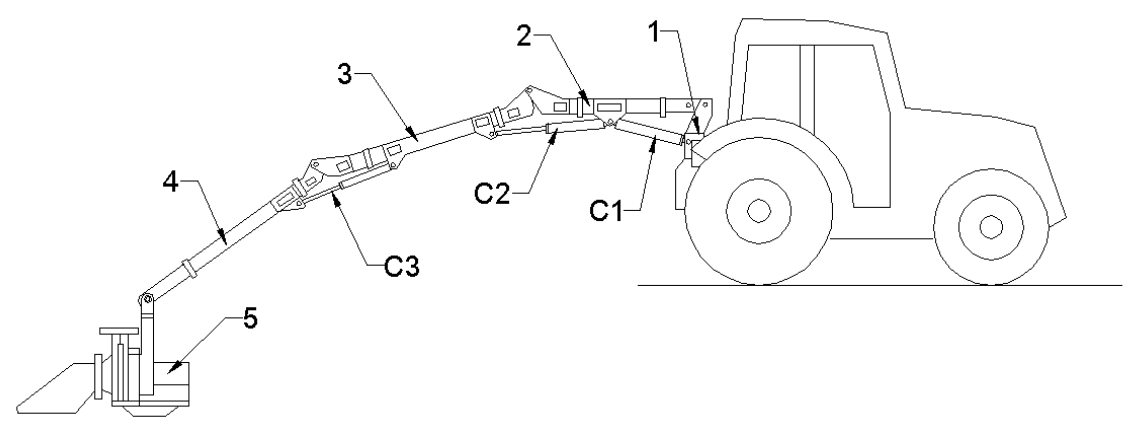

Figura 4. Modelo simplificado de mecanismo plano del sistema móvil

Debido a que el sistema de bombeo debe captar el agua desde fuentes con un nivel de agua menor o igual a $40 \mathrm{~cm}$, es necesario lograr una geometría en la admisión de la bomba que permita el mínimo arrastre de partículas desde la superficie sumergida.

Un modelo de tubería de succión con un prefiltro en un sistema de bombeo para conducir agua superficial de ríos, lagos y canales fue propuesto y simulado computacionalmente (Muschiatto y otros, 2015). El modelo computacional, consideró un canal poco 
profundo $(400 \mathrm{~mm})$ y pendiente cero, donde está sumergido el sistema de bombeo de succión de prefiltro, cuya forma tridimensional es cilíndrica, con un diámetro 1000 mm y altura $100 \mathrm{~mm}$, aunque el modelo bidimensional solo puede ver una sección transversal rectangular del mismo. Su forma y dimensiones fueron propuestas a fin de disminuir la velocidad de flujo en su entrada y así reducir el arrastre de las partículas del lecho de canal hacia la tubería de succión. En la succión se deberá tener una velocidad baja, para evitar recoger sedimentos, plantas, partículas grandes del fondo pudiendo reducir el flujo. Para los suministros rurales, las velocidades adecuadas están en el rango de 1,2 y 1,8 m/s, no obstante, se adopta como velocidad de entrada un valor aún menor. Perpendicular al canal se encuentra la primera porción del tubo de succión acoplado al prefiltro; con diámetro interior de $140 \mathrm{~mm}$.

El caudal teórico que ingresa a la tubería principal es un dato obtenido a partir del diseño agronómico. Básicamente, la descarga total requerida para el sistema en una aplicación de riego en particular es

$$
\mathrm{Q}_{\mathrm{T}}=\frac{10 \mathrm{Axd}}{\mathrm{t}}
$$

Donde $Q_{T}=$ caudal total teórico en $\mathrm{m}^{3} / \mathrm{h}, A=$ superficie a regar en ha; $t=$ tiempo de operación para la irrigación en h; $d=$ lámina de agua a aplicar, en $\mathrm{mm}$.

A fin de que los emisores funcionen correctamente, el sistema de riego debe tener en su entrada un valor de presión tal que, luego de producirse las disminuciones de presión debido a la fricción por la conducción del agua, los mismos tengan disponible en su entrada la presión de trabajo que indica el fabricante. La presión de proyecto o diseño, es la presión del agua a la entrada del cabezal de riego para lograr entonces un correcto funcionamiento del sistema. La presión $\mathrm{P}_{0}$ a la entrada se define como la necesaria para que la presión media de los emisores sea la que vierta el caudal de diseño. La misma se calcula teniendo en cuenta la altura de presión nominal del aspersor, la altura de pérdida total y el desnivel en el conducto (Dean et al., 2010).

\section{DISCUSIÓN}

Los resultados de diseño conceptual corroboran la utilidad de mantener como guía heurística la hipótesis analógica de Ernst Kapp, la cual complementada con los métodos de análisis de función, la matriz morfológica y el empleo de la información existente de configuraciones básicas de mecanismos, coetáneos al diseño del implemento, de un brazo robótico poliarticulado, permiten visualizar un conjunto factible de alternativas de diseño. 


\section{CONCLUSIONES}

El diseño de implementos de máquinas agrícolas es una actividad interdisciplinaria que involucra la obtención y aplicación de varios tipos de conocimientos especializados de manera de satisfacer los requerimientos del cliente e implica la utilización de diferentes métodos durante el proceso de diseño.

Se presentaron métodos para exploración y generación de diseños alternativos, utilizados en el diseño de un implemento agrícola polivalente, donde con la misma función técnica puede tener usos alternativos o diferentes funciones asignadas por el usuario, por ejemplo, la función de evacuar zonas anegadas. Se obtuvo la representación de análisis de función y una matriz morfológica o matriz de alternativas. Las mismas documentan en forma básica, la descomposición del sistema en diferentes tipos de sub funciones y las alternativas dentro de cada tipo de elemento, así como la lógica de integración o síntesis que impulsará qué configuraciones del sistema son realizables para poder ser aplicado como implemento a una máquina motriz agrícola.

En cuanto a la sostenibilidad ecológica de los arroyos o áreas de aguas superficiales se respeta la misma diseñando un implemento agrícola que se adapte a la altimetría típica del terreno y no a la inversa, esto es, se evita producir alteraciones físicas en los puntos de captación del agua superficial.

\section{AGRADECIMIENTOS}

Estas investigaciones fueron financiadas por la Sección de Ciencia y Técnica de la Universidad Nacional de Río Cuarto (U.N.R.C.) en el marco del proyecto Diseño paramétrico de un sistema mecánico móvil de bombeo de aguas superficiales utilizando síntesis morfológica, incluido en el programa Avances en el diseño e integración de dispositivos fluido mecánicos y de telecomunicaciones con aplicación a Sistemas Agroalimentarios. Se agradece al Ing. Guillermo Muschiatto por el aporte realizado en trabajo previo referenciado.

\section{REFERENCIAS BIBLIOGRÁFICAS}

Cross, Nigel. 1996. Engineering Design Methods. Strategies for Design Products. John Wiley\&Sons

Dean, Raúl; Maglione, Livio y Elaskar, Sergio. 2009. «Una Perspectiva Transdisciplinaria de un Programa de Investigación sobre Riego Complementario por Aspersión». Avances en Ingeniería Rural 2007-2009. Edit. N. Di Leo y otros. Universidad Nacional de Rosario. 477-483. 
Dean, Raúl; Maglione Livio y Pianucci Agustín. 2010. Aspectos Básicos del Diseño Hidráulico de un Sistema de Riego por Aspersión de Avance Frontal. Segundo Congreso Argentino de Ingeniería Mecánica II CAIM 2010 - San Juan - Noviembre 2010 .

Dresch, Aline; Lacerda, Daniel y Antunes, José. 2015. Design Science Research: A Method for Science and Technology Advancement. Springer.

Finger, S.; Dixon, J. 1989a. «Part I: Descriptive, Prescriptive, and Computer - Based Models of Design Process». Res Eng Des. Vol 1. 51-67

Finger S.; Dixon, J. 1989b. «A Review of Research in Mechanical Engineering Design. Part II: Representations, Analysis, and Design for the Life Cycle». Res Eng Des. Vol 1. 121-137.

Kapp, Ernest. 1877. «Líneas fundamentales de una filosofía de la técnica: acerca de la historia del surgimiento de la cultura desde nuevos puntos de vista», Braunscheweig, Westermann. En: Tecnos Vol. XVII N 3. 1998

Muschiatto, Guillermo; Maglione, Livio y Dean, Raúl. 2015. Advances in the Design of Shallow Water Pumping System for Mobile Use at the Central Region of Argentina. 23rd ABCM International Congress of Mechanical Engineering December 6-11, RJ, Brazil

Salinas, A. et al. 2006. «Riego Suplementario en Cultivos Extensivos en la Provincia de Córdoba Situación Actual». EEA Manfredi. INTA. Manfredi. Córdoba.

Recepción: 19/01/2018

Aceptación: 19/06/2018

\section{Correspondencia}

Raúl Alberto Dean

raulalbertodean@gmail.com 